\title{
EFFECTIVENESS OF DENTAL TRAUMATIC INJURIES' EDUCATIONAL MESSAGE ON SCHOOL TEACHERS IN BEIRUT-CLUSTER RANDOMIZED FIELD TRIAL
}

\author{
Carolina W. Yordi*, Sherine B.Y. Badr ${ }^{* *}$ and Mohamed Shokry***
}

\begin{abstract}
Introduction: School teachers are most likely to be involved at the site of the injuries, making their knowledge of emergency management fundamental to the provision of an appropriate care to children and adolescents.
\end{abstract}

Aim of the study: To assess the effectiveness of educational message on the knowledge and practice of school teachers regarding dental trauma.

Materials and Methods: Cluster Randomized Controlled Trial. Schools of Beirut were divided into three categories (public, semi private and private). Two-hundred teachers of each category were selected randomly and divided into "Test and Control group". An educational message, using power point presentation, brochures and posters, was implemented to teachers in test group. Immediate and long term assessment were performed to evaluate the effect of the educational program.

Results: Immediately after the implementation of the educational message, there was a significant improvement in the knowledge and practice of teachers of the test group, persisting approximately the same six months later.

Conclusion: The implementation of a dental health educational program using more than one modality, proved to be effective in improving the knowledge of school teachers who are the first individuals to face dental trauma in schools.

KEY WORDS: Dental trauma, Tooth injuries, School teachers, Knowledge, Educational tools.

* BDS, Department of Pediatric Dentistry, Faculty of Dentistry, Beirut Arab University, Lebanon.

** BDS, MSc, PhD, DPHE, Associate Professor of Pediatric Dentistry, Faculty of Dentistry, Beirut Arab University, Lebanon, Faculty of Oral and Dental Medicine, Cairo University, Egypt.

*** BDS, MSc, PhD, Maxillofacial Surgery, Faculty of Dentistry, Beirut Arab University, Lebanon, Lecturer of Oral and Maxillofacial Surgery, Faculty of Dentistry, Alexandria University, Egypt. 


\section{INTRODUCTION}

Oral and dental health education must evolve to promote organized community efforts to prevent and control disease prevalence. All dental professionals will require continued updating of knowledge and practice, in order to create supportive environments, strengthen community action, develop personal skills and re-orientate health services in the pursuit of oral health goals ${ }^{(1)}$, such as the provision of preventive programs delivered in a variety of settings.

According to the World Health Organization, one of the serious public health problems among children and adolescents throughout the world are DTI and can vary from minor enamel fractures to extensive maxillofacial damage involving the supporting structures and displacement or avulsion of teeth having significant functional, esthetic, and psychological negative effects on children ${ }^{(2,3)}$. Treatment in such cases can be complicated, expensive and sometimes lengthy ${ }^{(2,4-6)}$.

The prevalence rates of $5-12 \%$ were found in children aged 6-12 years in the Middle East and also in young children with less than three years of age, due to their immature motor coordination, being more predisposed to falls. Other studies reported that more than $20 \%$ of all school children experienced dental trauma, with most of these injuries occurring before age nineteen ${ }^{(5,7,8)}$. Studies from Brazil showed that the prevalence of dental trauma in preschool is about $40 \%$ and in school it varies from $7.8 \%$ to $35.8 \%{ }^{(9)}$. Some studies assert that the number of cases with dental trauma will exceed dental caries or periodontal problems resulting in high costs to Public Health Services ${ }^{(10,11)}$

Since children spend much of their time in schools, teachers form the group who commonly supervise their physical activity and play an important role in managing these injuries, providing good care and improving its prognosis $(12,13)$.
However, multiple studies have demonstrated that these individuals have relatively poor knowledge of this topic ${ }^{(14-17)}$. This should not be underestimated, particularly because school is a place under surveillance. Therefore, educational staff should master emergency measures to help, calm children down and minimize accident sequela ${ }^{(18,19)}$.

Various methods may be used to improve knowledge of school teachers, including educational brochures and posters, lectures, courses, seminars, and regular visits of dentists to schools. Furthermore, to achieve adequate awareness, educative and motivational programs can be planned, developed, and organized in schools such as the use of seminars and lectures during formal and continuing education of teachers ${ }^{(16)}$.

Multiple international studies conducted in Iran, India, Turkey and other countries have implemented educational programs in order to raise the awareness regarding DTI among school teachers ${ }^{(15,18,20-22)}$.

Actually, in Lebanon no dental health educational program concerning DTI has been implemented targeting school teachers. For this reason, this study was conducted to carry out effective educational program aimed to raise the awareness and reduce the incidence and consequences of DTI.

\section{AIM OF THE STUDY}

To assess the effectiveness of educational message on the knowledge and practice of school teachers regarding dental trauma.

\section{MATERIALS AND METHODS}

\section{Study Design}

Cluster Randomized Field Trial.

\section{Study Setting}

The study was conducted on school teachers in Beirut- Lebanon belonging to public, semi private and private schools. 


\section{Study Sampling}

The list of schools was obtained from the Ministry of Education (UNESCO). Schools of Beirut were divided into three categories: public, semi private and private. Two-hundred teachers from each category were randomly selected from those who accepted to participate in the study and signed the consent form, with respect to confidentiality and anonymity. Finally, 600 teachers were included in this study. Each school category was divided into two groups: "Test and Control" each of 100 teachers.

Teachers were not informed of the identity of the group they belong to (Test/Control), educational material that they were going to receive and the duration of the trial ${ }^{(23)}$. Pregnant women and assistant/substitute teachers were excluded from the study to prevent the risk of withdrawal throughout the implementation of the trial.

\section{Questionnaire}

Questionnaires in Arabic and English versions were constructed and distributed to the teachers and later collected by the investigator immediately after completion. The questionnaires were divided into three sections: The first section contained basic information such as socio-demographic details including: gender, age, level of education (high school or superior) and seniority (duration of work in the educational field). The second section identified whether the respondents received prior information concerning DTI or had previously experienced any case of dental trauma. The third section was referred to the knowledge and practice of DTI ${ }^{(12,13,23)}$. Fig. (1)

\section{Power point presentation, Brochure and Poster}

The dental educational presentation, brochures and posters were very specific, colourful and with informative pictures. Brochures and posters were created in English and French to facilitate the understanding for all teachers involved, whether they were, English or French educated. A pilot study was conducted to test the questionnaire validity.

\section{Implementation of the trial}

The trial was applied in four steps for the "Test Group". During the first step, teachers were asked to fill out a questionnaire in 20 minutes time. Secondly, they received an educational message using a power point presentation in order to improve their awareness level regarding DTI. During the third step, the same questionnaire was redistributed after the presentation to assess the immediate effect of the educational message ${ }^{(24)}$. Brochures were given to all teachers with the most relevant information regarding the topic. Moreover, a poster was delivered to each school visited. The last step of the study was applied after six months by redistributing the same questionnaire to assess the long term effect of the educational program.

The "Control Group" however, received the same questionnaire twice. Once at the beginning of the study and finally, after 6 months, without the implementation of any dental educational message in order to verify if there is a difference between the knowledge and practice of the two groups.

At the end of the trial, brochures and posters were also given to the teachers belonging to the control group in order to benefit them from the study.

At the end of the trial, brochures and posters were also given to the teachers belonging to the Control group in order to raise their awareness regarding DTI as well.

\section{Data analysis}

Data collected was qualitative, so it was presented in form of numbers and percentages.

Statistical analyses were performed using SPSS software for windows version 18.0. The statistical significance level was set at $\mathrm{p}<0.05$. Chi square test and Fisher Exact test were used to compare the knowledge and practice between different groups (public, semi private and private schools). Chi square test for trend was carried out to assess the percentage of true answers for each question over time. 
NAME:

LAST NAME:

PHONE NUMBER:

CHOOSE THE MOST APPROPIATE ANSWER. ONLY ONE OPTION IS ALLOWED.

SECTION A:

C. KNOWLEDGE AND PRACTICE

\begin{tabular}{|l|l||}
\hline A1: GENDER & $\begin{array}{l}\text { A4: LEVEL OF } \\
\text { EDUCATION } \\
\text { O Male }\end{array}$ \\
O Figh School \\
O Superior \\
A2: AGE
\end{tabular}

SECTION B: PREVIOUS DENTAL TRAUMA EXPERIENCE

B1: HAVE YOU EVER RECEIVED ANY INFORMATION RELATED TO TRAUMATIC INJURIES:

Y Yes

No

B2: IF YES, MENTION THE SOURCE OF INFORMATION:

First aid courses

Education in pedagogic faculty

Health Practitioner

Other sources. Specify:

B3: ARE YOU CONFIDENT

DISTINGUISHING TYPE OF TEETH

(PRIMARY AND PERMANENT):

O Yes

No

B4: WHAT DO WE MEAN BY DENTAL

TRAUMATIC INJURIES:

$O$ Caries in the tooth

Violent knock on the teeth

Tooth ache

$O$ Do not know

B5: HAVE YOUEVER EXPERIENCED

ANY CASE OF DENTAL TRAUMA IN

YOUR SCHOOL:

$\mathrm{O}$ Yes

No

C1: DESCRIBE A SITUATION THAT COULD RESULT IN DENTAL TRAUMA AT THE SCHOOL SETTING:

Sport activities

$O$ Falls during walking or sports

As a result of an object impact to the mouth

$O$ Due to a conflict between students

All of the above

None of the above. Specify:

C2: IMMEDIATE MANAGEMENT OF FRACTURED TEETH:

The fractured part is useless, ignore it

Try to find the fractured part, wrap it with gauze or tissue and bring it for examination and treatment $\bigcirc$ Put it in liquid medium and bring it for examination and treatment

Other. Specify:

Do not know

C3: IMMEDIATE MANAGEMENT OF DISPLACED TEETH:

$\mathrm{Do}$ not touch, let it remains in its new position

$\bigcirc$ Try to put back to the original position

Oother. Specify:

\section{$\bigcirc$ Do not know}

C4: WHAT WOULD YOU DO WITH THE CHILD IN CASE OF A KNOCKEDOUT TOOTH:

$O$ Inform the family and transfer the child to a dentist

Would contact the hospital

Would not do anything, leave the child at school

$\bigcirc$ Would contact by phone a dentist, inform about the incident and listen

to his/her advice

Other. Specify:

Do not know
C5: SHOULD KNOCKED- OUT BABY (PRIMARY) TEETH BE PUT BACK TO ORIGINAL POSITION:

$O$ Yes

No

Do not know

C6: SHOULD KNOCKED- OUT

PERMANENT TEETH BE PUT BACK TO ORIGINAL POSITION:

$\mathrm{O}$ Yes

No

Do not know

C7: IF YOU COULD FIND THE TOOTH, HOW WOULD YOU HOLD IT?:

$O$ By the crown

By the root

Anyway- It does not make any difference

$O$ I would leave it.

C8: IF YOU WITNESS A CASE OF KNOCKED-OUT TOOTH WHAT WOULD IT BE YOUR APPROACH:

The tooth is useless, ignore it $\mathrm{Rub}$ it vigorously to remove all the dirt

Wash it with tap water

OPlace Immediately

Store it in a proper medium and seek dental clinic immediately

Other. Specify:

C9: MEDIUM FOR STORING THE AVULSED TOOTH (if it would be found):

Place it in clean gauze or tissue

$O$ Place it in milk

Place it in physiological saline

Place it in patient's saliva

Place it in distilled water

Place it in disinfectant solution

Other. Specify:

Do not know

C10: HAVE YOU EVER HEARD OF ANYTHING THAT CAN BE PUT IN THE MOUTH TO PROTECT PERMANENT TEETH WHILE PRACTICING SPORTS: $\mathrm{O}$ Yes No

Figure 1: Questionnaire Sample in English version 
Ethical approval before the implementation of the trial was obtained from the concerned authorities: Institutional Review Board (IRB) Beirut Arab University (code: 2015H-0021-D-M-0081), Minister of Education "UNESCO", Minister of Health and the concerned authorities from the selected schools. Moreover, consent forms were signed by each one of the teachers.

\section{RESULTS}

\section{Socio-demographic characteristics}

Socio-demographic characteristics of the teachers were significantly related to the school group. In private schools, there were significantly more females: 195 (97.5\%) compared to semi private and private schools, 191 (95.5\%) and 177 (88.5\%) respectively. Significantly, more young teachers were found in semi private and private schools with a number of 115 (57.5\%) and 103 (51.5\%) respectively, compared to public school teachers: $70(35.0 \%)$. Moreover, a significant difference was found regarding nationality, $200(100 \%)$ teachers from public and semi private schools were Lebanese, contrary to teachers belonging to private schools, where 16 (8.0\%) were Non-Lebanese. Furthermore, there were significantly more teachers with superior education (university degree) in private schools: 181 (90.5\%), compared to semi private and public school teachers: $163(81.5 \%)$ and $159(79.5 \%)$ respectively. Also, private school teachers showed significantly more duration of work in the educational field (seniority): 86 (43\%) compared to semi private and public school teachers: $74(37 \%)$ and $75(37.5 \%)$ respectively. Table (1)

\section{Dental Trauma experience}

Before the power point presentation, teachers from the test group; 18(18.0\%), 15(15.0\%) and $14(14.0 \%)$ and those from the control group; 14 $(14.0 \%), 8(8.0 \%)$ and $14(14.0 \%)$ belonging to public, semi private and private schools respectively, received information related to traumatic injuries prior to being enrolled in the present study. Table (2)

In addition, 65(65.0\%), 47(47.0\%) and $59(59.0 \%)$ teachers from the test group and $45(45.0 \%), 48(48.0 \%)$ and $65(65.0 \%)$ teachers from the control group belonging to public, semi private and private schools respectively, were confident in distinguishing type of teeth (primary and permanent) before the implementation of the educational program. Table (3)

Moreover, 70(70.0\%), 75(75.0\%) and 64(64.0\%) teachers from the test group; and 68(68.0\%), $66(66.0 \%)$ and $78(78.0 \%)$ teachers from the control group belonging to public, semi private and private schools respectively, gave the correct answer regarding DTI definition, which are recognized as a violent knock on the teeth. Table (4)

Immediately after the presentation, the percentages had significantly increased in teachers belonging to the test group (p-value $<0.001$ ) and remained the same six months later. However, the correct answers of the control group did not increase in the period of six months.

\section{Knowledge and practice}

Concerning the immediate management of fractured teeth, 22(22.0\%), 16(16.0\%) and $24(24.0 \%)$ teachers from the test group; and $14(14.0 \%), 12(12.0 \%)$ and $12(12.0 \%)$ from the control belonging to public, semi private and private schools respectively, gave the correct answer regarding the immediate management of fractured teeth which is: "Put the fractured part in liquid medium and seek dental consultation for examination and treatment". Table (5)

Relating to the immediate management of displaced teeth, 32(32.0\%), 11(11.0\%) and 16(16\%) teachers from the test group; and 25(25.0\%), $16(16.0 \%)$ and $22(22.0 \%)$ from the control group 
belonging to public, semi private and private schools respectively, gave the correct answer regarding the immediate management of displaced teeth which is: "Try to put back to the original position". Table (6)

Regarding the immediate management of avulsed primary teeth, 47(47.0\%), 39(39.0\%) and $39(39.0 \%)$ teachers from the test group and; $47(47.0 \%), 35(35.0 \%)$ and $36(36.0 \%)$ from the control group belonging to public, semi private, and private schools respectively, believed that they should not put back knocked-out primary teeth to their original position which is the correct answer. Table (7)

With reference to the replantation of avulsed permanent teeth, 49(49.0\%), 29(29.0\%) and $39(39.0 \%)$ teachers from the test group and; $34(34.0 \%), 36(36.0 \%)$ and $29(29.0 \%)$ teachers from the control group belonging to public, semi private and private schools respectively, assumed that they should put back knocked-out permanent teeth to their original position which is the correct answer. Table (8)

As regards to the handling of avulsed permanent teeth, $55(55 \%), 46(46 \%)$ and 52(52\%) teachers from the test group; and 46(46.0\%), 48(48.0\%) and $43(43.0 \%)$ from the control group belonging to public, semi private and private schools respectively, stated that they would hold the tooth by the crown which is the correct answer. Table (9)

Concerning the immediate Replantation of avulsed permanent teeth, $8(8.0 \%), 7(7.0 \%)$ and $8(8.0 \%)$ teachers from the test group; and $7(7.0 \%)$, $8(8.0 \%)$ and $6(6.0 \%)$ from the control group, belonging to public, semi private and private schools respectively, thought that they should place the avulsed permanent tooth immediately. Table (10)

Regarding the delayed Replantation, 36(36.0\%), $32(36.0 \%)$ and $29(29.0 \%)$ teachers from the test group; and 47(47.0\%), 31(31.0\%) and 45(45.0\%) from the control group belonging to public, semi private and private schools respectively, stated that they would store the knocked out teeth in a proper medium and seek a dental clinic immediately. Table (11)

Concerning the recommended mediums for storing the avulsed permanent tooth, 29(29.0\%), $14(14.0 \%)$ and $27(27.0 \%)$ teachers from the test group; and 13(13.0\%), 20(20.0\%) and 22(22.0\%) from the control group belonging to public, semi private and private schools respectively, gave the correct answer. Table (12)

Referring to the knowledge regarding mouth guards, $69(69.0 \%), \quad 53(53.0 \%)$ and $74(74.0 \%)$ teachers from the test group; and 66(66.0\%), $73(73.0 \%)$ and $68(68.0 \%)$ from the control group belonging to public, semi private and private schools respectively, heard of something that can be put in the mouth to protect the teeth while practicing sports. Table (13)

Regarding all the questions concerning knowledge and practice of DTI, the percentages significantly increased in the test group immediately after the power point presentation, observing an insignificant decrease six months later. On the contrary, no significant difference was found in the control group six months later. Table (5-13) 
TABLE (1) Socio-demographic characteristics of the participants according to school group.

\begin{tabular}{|c|c|c|c|c|}
\hline & \multicolumn{3}{|c|}{ SCHOOL GROUPS } & \multirow[b]{2}{*}{ p-value } \\
\hline & $\begin{array}{l}\text { Public } \\
\text { N=200 }\end{array}$ & $\begin{array}{c}\text { Semi private } \\
\quad \mathrm{N}=\mathbf{2 0 0}\end{array}$ & $\begin{array}{l}\text { Private } \\
\mathrm{N}=\mathbf{2 0 0}\end{array}$ & \\
\hline \multicolumn{5}{|l|}{ Gender } \\
\hline Male & $23(11.5 \%)$ & $9(4.5 \%)$ & $5(2.5 \%)$ & \multirow{2}{*}{$0.001^{*}$} \\
\hline Female & $177(88.5 \%)$ & $191(95.5 \%)$ & $195(97.5 \%)$ & \\
\hline \multicolumn{5}{|l|}{ Age } \\
\hline 20-40 years & $70(35.0 \%)$ & $115(57.5 \%)$ & $103(51.5 \%)$ & \multirow{3}{*}{$0.001 *$} \\
\hline 41-60 years & $97(48.5 \%)$ & $83(41.5 \%)$ & $84(42.0 \%)$ & \\
\hline 61years or more & $33(16.5 \%)$ & $2(1.0 \%)$ & $13(6.5 \%)$ & \\
\hline \multicolumn{5}{|l|}{ Nationality } \\
\hline Lebanese & $200(100.0 \%)$ & $200(100.0 \%)$ & $184(92.0 \%)$ & \multirow{2}{*}{$0.001 *$} \\
\hline Non Lebanese & $0(.0 \%)$ & $0(.0 \%)$ & $16(8.0 \%)$ & \\
\hline \multicolumn{5}{|l|}{ Education } \\
\hline High school & $41(20.5 \%)$ & $37(18.5 \%)$ & $19(9.5 \%)$ & \multirow{2}{*}{$0.006^{*}$} \\
\hline Superior education & $159(79.5 \%)$ & $163(81.5 \%)$ & $181(90.5 \%)$ & \\
\hline \multicolumn{5}{|l|}{ Seniority } \\
\hline$<1$ year & $4(2.0 \%)$ & $10(5.0 \%)$ & $2(1.0 \%)$ & \multirow{5}{*}{$0.043 *$} \\
\hline $1-5$ years & $29(14.5 \%)$ & $43(21.5 \%)$ & $30(15.0 \%)$ & \\
\hline 6-10 years & $28(14.0 \%)$ & $31(15.5 \%)$ & $30(15.0 \%)$ & \\
\hline $11-15$ years & $64(32.0 \%)$ & $42(21.0 \%)$ & $52(26.0 \%)$ & \\
\hline 16 and above & $75(37.5 \%)$ & $74(37.0 \%)$ & $86(43.0 \%)$ & \\
\hline
\end{tabular}

*The statistical significance level was set at $p<0.05$; Chi- Square test

TABLE (2) Information received related to traumatic injuries.

\begin{tabular}{|c|c|c|c|c|c|}
\hline \multirow{2}{*}{ School Groups } & \multirow{2}{*}{\multicolumn{2}{|c|}{$\begin{array}{l}\text { Have you ever received any information } \\
\text { related to traumatic injuries? }\end{array}$}} & \multicolumn{2}{|c|}{ Time } & \multirow{2}{*}{ p-value } \\
\hline & & & Before & 6 months later & \\
\hline \multirow{4}{*}{ Public } & \multirow{2}{*}{ Control } & Yes & $14(14.0 \%)$ & $14(14.0 \%)$ & \multirow{2}{*}{1.000} \\
\hline & & No & $86(86.0 \%)$ & $86(86.0 \%)$ & \\
\hline & \multirow{2}{*}{ Test } & Yes & $18(18.0 \%)$ & $100(100.0 \%)$ & \multirow{2}{*}{$<0.001^{*}$} \\
\hline & & No & $82(82.0 \%)$ & $0(.0 \%)$ & \\
\hline \multirow{4}{*}{ Semi Private } & \multirow{2}{*}{ Control } & Yes & $8(8.0 \%)$ & $8(8.0 \%)$ & \multirow{2}{*}{1.000} \\
\hline & & No & $92(92.0 \%)$ & $92(92.0 \%)$ & \\
\hline & \multirow{2}{*}{ Test } & Yes & $15(15.0 \%)$ & $100(100.0 \%)$ & \multirow{2}{*}{$<0.001^{*}$} \\
\hline & & No & $85(85.0 \%)$ & $0(.0 \%)$ & \\
\hline \multirow{4}{*}{ Private } & \multirow{2}{*}{ Control } & Yes & $14(14.0 \%)$ & $14(14.0 \%)$ & \multirow{2}{*}{1.000} \\
\hline & & No & $86(86.0 \%)$ & $86(86.0 \%)$ & \\
\hline & \multirow{2}{*}{ Test } & Yes & $14(14.0 \%)$ & $100(100.0 \%)$ & \multirow{2}{*}{$<0.001 *$} \\
\hline & & No & $86(86.0 \%)$ & $0(.0 \%)$ & \\
\hline
\end{tabular}

\footnotetext{
*The statistical significance level was set at $p<0.05 ;$ Chi- Square test for trend
} 
TABLE (3) Type of dentition (primary and permanent).

\begin{tabular}{|c|c|c|c|c|c|c|}
\hline \multirow{2}{*}{$\begin{array}{l}\text { School } \\
\text { Groups }\end{array}$} & \multirow{2}{*}{\multicolumn{2}{|c|}{$\begin{array}{l}\text { Are you confident } \\
\text { Distinguishing type of } \\
\text { teeth? }\end{array}$}} & \multicolumn{3}{|c|}{ Time } & \multirow[b]{2}{*}{ p-value } \\
\hline & & & Before & Immediate after & 6 months later & \\
\hline \multirow{4}{*}{ Public } & \multirow{2}{*}{ Control } & Yes & $45(45.0 \%)$ & & $46(46.0 \%)$ & \multirow{2}{*}{0.887} \\
\hline & & No & $55(55.0 \%)$ & & $54(54.0 \%)$ & \\
\hline & \multirow{2}{*}{ Test } & Yes & $65(65.0 \%)$ & $99(99.0 \%)$ & $99(99.0 \%)$ & \multirow{2}{*}{$<0.001^{*}$} \\
\hline & & No & $35(35.0 \%)$ & $1(1.0 \%)$ & $1(1.0 \%)$ & \\
\hline \multirow{4}{*}{$\begin{array}{c}\text { Semi } \\
\text { Private }\end{array}$} & \multirow{2}{*}{ Control } & Yes & $48(48.0 \%)$ & & $48(48.0 \%)$ & \multirow{2}{*}{1.000} \\
\hline & & No & $52(52.0 \%)$ & & $52(52.0 \%)$ & \\
\hline & \multirow{2}{*}{ Test } & Yes & $47(47.0 \%)$ & $92(92.0 \%)$ & $92(92.0 \%)$ & \multirow{2}{*}{$<0.001 *$} \\
\hline & & No & $53(53.0 \%)$ & $8(8.0 \%)$ & $8(8.0 \%)$ & \\
\hline \multirow{3}{*}{ Private } & \multirow{2}{*}{ Control } & Yes & $65(65.0 \%)$ & & $65(65.0 \%)$ & \multirow{2}{*}{1.000} \\
\hline & & No & $35(35.0 \%)$ & & $35(35.0 \%)$ & \\
\hline & Test & Yes & $59(59.0 \%)$ & $91(91.0 \%)$ & $91(91.0 \%)$ & $<0.001 *$ \\
\hline
\end{tabular}

* The statistical significance level was set at $p<0.05$; Chi-Square test for trend

TABLE (4) Definition of DTI.

\begin{tabular}{|c|c|c|c|c|c|c|}
\hline \multirow{2}{*}{$\begin{array}{l}\text { School } \\
\text { Groups }\end{array}$} & \multirow{2}{*}{\multicolumn{2}{|c|}{$\begin{array}{c}\text { What do we mean by Dental } \\
\text { Traumatic Injuries? }\end{array}$}} & \multicolumn{3}{|c|}{ Time } & \multirow{2}{*}{ p-value } \\
\hline & & & Before & Immediate after & Six months later & \\
\hline \multirow{4}{*}{ Public } & \multirow{2}{*}{ Control } & True answer & $68(68.0 \%)$ & & $70(70.0 \%)$ & \multirow{2}{*}{0.760} \\
\hline & & False answers & $32(32.0 \%)$ & & $30(30.0 \%)$ & \\
\hline & \multirow{2}{*}{ Test } & True answer & $70(70.0 \%)$ & $100(100.0 \%)$ & $100(100.0 \%)$ & \multirow{2}{*}{$<0.001 *$} \\
\hline & & False answers & $30(30.0 \%)$ & $0(.0 \%)$ & $0(.0 \%)$ & \\
\hline \multirow{4}{*}{$\begin{array}{c}\text { Semi } \\
\text { Private }\end{array}$} & \multirow{2}{*}{ Control } & True answer & $66(66.0 \%)$ & & $70(70.0 \%)$ & \multirow{2}{*}{0.544} \\
\hline & & False answers & $34(34.0 \%)$ & & $30(30.0 \%)$ & \\
\hline & \multirow{2}{*}{ Test } & True answer & $75(75.0 \%)$ & $100(100.0 \%)$ & $100(100.0 \%)$ & \multirow{2}{*}{$<0.001^{*}$} \\
\hline & & False answers & $25(25.0 \%)$ & $0(.0 \%)$ & $0(.0 \%)$ & \\
\hline \multirow{4}{*}{ Private } & \multirow{2}{*}{ Control } & True answer & $78(78.0 \%)$ & & $78(78.0 \%)$ & \multirow{2}{*}{1.000} \\
\hline & & False answers & $22(22.0 \%)$ & & $22(22.0 \%)$ & \\
\hline & \multirow{2}{*}{ Test } & True answer & $64(64.0 \%)$ & $100(100.0 \%)$ & $100(100.0 \%)$ & \multirow{2}{*}{$<0.001 *$} \\
\hline & & False answers & $36(36.0 \%)$ & $0(.0 \%)$ & $0(.0 \%)$ & \\
\hline
\end{tabular}

*The statistical significance level was set at $p<0.05 ;$ Chi-Square test for trend 
TABLE (5) Immediate management of Fractured Teeth.

\begin{tabular}{|c|c|c|c|c|c|c|}
\hline \multirow{2}{*}{$\begin{array}{l}\text { School } \\
\text { Groups }\end{array}$} & \multirow{2}{*}{\multicolumn{2}{|c|}{$\begin{array}{l}\text { "Put it in liquid medium and } \\
\text { seek dental consultation for } \\
\text { examination and treatment" }\end{array}$}} & \multicolumn{3}{|c|}{ Time } & \multirow[b]{2}{*}{ p-value } \\
\hline & & & Before & Immediate after & Six months later & \\
\hline \multirow{4}{*}{ Public } & \multirow{2}{*}{ Control } & True answer & $14(14.0 \%)$ & & $17(17.0 \%)$ & \multirow{2}{*}{0.558} \\
\hline & & False answer & $86(86.0 \%)$ & & $83(83.0 \%)$ & \\
\hline & \multirow{2}{*}{ Test } & True answer & $22(22.0 \%)$ & $90(90.0 \%)$ & $84(84.0 \%)$ & \multirow{2}{*}{$<0.001 *$} \\
\hline & & False answer & $78(78.0 \%)$ & $10(10.0 \%)$ & $16(16.0 \%)$ & \\
\hline \multirow{4}{*}{$\begin{array}{c}\text { Semi } \\
\text { Private }\end{array}$} & \multirow{2}{*}{ Control } & True answer & $12(12.0 \%)$ & & $12(12.0 \%)$ & \multirow{2}{*}{1.000} \\
\hline & & False answer & $88(88.0 \%)$ & & $88(88.0 \%)$ & \\
\hline & \multirow{2}{*}{ Test } & True answer & $16(16.0 \%)$ & $93(93.0 \%)$ & $91(91.0 \%)$ & \multirow{2}{*}{$<0.001 *$} \\
\hline & & False answer & $84(84.0 \%)$ & $7(7.0 \%)$ & $7(7.0 \%)$ & \\
\hline \multirow{4}{*}{ Private } & \multirow{2}{*}{ Control } & True answer & $12(12.0 \%)$ & & $12(12.0 \%)$ & \multirow{2}{*}{1.000} \\
\hline & & False answer & $88(88.0 \%)$ & & $88(88.0 \%)$ & \\
\hline & \multirow{2}{*}{ Test } & True answer & $24(24.0 \%)$ & $95(95.0 \%)$ & $90(90.0 \%)$ & \multirow{2}{*}{$<0.001 *$} \\
\hline & & False answer & $76(76.0 \%)$ & $5(5.0 \%)$ & $10(10.0 \%)$ & \\
\hline
\end{tabular}

* The statistical significance level was set at $p<0.05$; Chi-Square test for trend

TABLE (6) Immediate management of Displaced Teeth.

\begin{tabular}{|c|c|c|c|c|c|c|}
\hline \multirow{2}{*}{ School Groups } & \multirow{2}{*}{\multicolumn{2}{|c|}{$\begin{array}{l}\text { "Try to put back to the } \\
\text { original position" }\end{array}$}} & \multicolumn{3}{|c|}{ Time } & \multirow{2}{*}{ p-value } \\
\hline & & & Before & Immediate after & Six months later & \\
\hline \multirow{4}{*}{ Public } & \multirow{2}{*}{ Control } & True answer & $25(25.0 \%)$ & & $25(25.0 \%)$ & \multirow{2}{*}{0.871} \\
\hline & & False answer & $75(75.0 \%)$ & & $75(75.0 \%)$ & \\
\hline & \multirow{2}{*}{ Test } & True answer & $30(30.0 \%)$ & $91(91.0 \%)$ & $86(86.0 \%)$ & \multirow{2}{*}{$<0.001 *$} \\
\hline & & False answer & $70(70.0 \%)$ & $9(9.0 \%)$ & $14(14.0 \%)$ & \\
\hline \multirow{4}{*}{ Semi Private } & \multirow{2}{*}{ Control } & True answer & $16(16.0 \%)$ & & $16(16.0 \%)$ & \multirow{2}{*}{1.000} \\
\hline & & False answer & $84(84.0 \%)$ & & $84(84.0 \%)$ & \\
\hline & \multirow{2}{*}{ Test } & True answer & $11(11.0 \%)$ & $95(95.0 \%)$ & $92(92.0 \%)$ & \multirow{2}{*}{$<0.001^{*}$} \\
\hline & & False answer & $89(89.0 \%)$ & $5(5.0 \%)$ & $8(8.0 \%)$ & \\
\hline \multirow{4}{*}{ Private } & \multirow{2}{*}{ Control } & True answer & $22(22.0 \%)$ & & $22(22.0 \%)$ & \multirow{2}{*}{1.000} \\
\hline & & False answer & $78(78.0 \%)$ & & $78(78.0 \%)$ & \\
\hline & \multirow{2}{*}{ Test } & True answer & $16(16.0 \%)$ & $98(98.0 \%)$ & $88(88.0 \%)$ & \multirow{2}{*}{$<0.001 *$} \\
\hline & & False answer & $84(84.0 \%)$ & $2(2.0 \%)$ & $12(12.0 \%)$ & \\
\hline
\end{tabular}

*The statistical significance level was set at $p<0.05$; Chi-Square test for trend 
TABLE (7) Immediate management of avulsed primary teeth.

\begin{tabular}{|c|c|c|c|c|c|c|}
\hline \multirow{2}{*}{$\begin{array}{l}\text { School } \\
\text { Groups }\end{array}$} & \multirow{2}{*}{\multicolumn{2}{|c|}{$\begin{array}{l}\text { "knocked- out primary teeth } \\
\text { should not be put back to their } \\
\text { original position" }\end{array}$}} & \multicolumn{3}{|c|}{ Time } & \multirow[b]{2}{*}{ p-value } \\
\hline & & & Before & Immediate after & Six months later & \\
\hline \multirow{4}{*}{ Public } & \multirow{2}{*}{ Control } & True answer & $47(47.0 \%)$ & & $45(45.0 \%)$ & \multirow{2}{*}{0.671} \\
\hline & & False answer & $53(53.0 \%)$ & & $55(55.0 \%)$ & \\
\hline & \multirow{2}{*}{ Test } & True answer & $47(47.0 \%)$ & $99(99.0 \%)$ & $86(86.0 \%)$ & \multirow{2}{*}{$<0.001^{*}$} \\
\hline & & False answer & $53(53.0 \%)$ & $1(1.0 \%)$ & $14(14.0 \%)$ & \\
\hline \multirow{4}{*}{$\begin{array}{c}\text { Semi } \\
\text { Private }\end{array}$} & \multirow{2}{*}{ Control } & True answer & $35(35.0 \%)$ & & $35(35.0 \%)$ & \multirow{2}{*}{1.000} \\
\hline & & False answer & $65(65.0 \%)$ & & $65(65.0 \%)$ & \\
\hline & \multirow{2}{*}{ Test } & True answer & $39(39.0 \%)$ & $100(100.0 \%)$ & $91(91.0 \%)$ & \multirow{2}{*}{$<0.001 *$} \\
\hline & & False answer & $61(61.0 \%)$ & $0(.0 \%)$ & $9(9.0 \%)$ & \\
\hline \multirow{4}{*}{ Private } & \multirow{2}{*}{ Control } & True answer & $36(36.0 \%)$ & & $39(39.0 \%)$ & \multirow{2}{*}{0.558} \\
\hline & & False answer & $64(64.0 \%)$ & & $61(61.0 \%)$ & \\
\hline & \multirow[b]{2}{*}{ Test } & True answer & $39(39.0 \%)$ & $96(96.0 \%)$ & $94(94.0 \%)$ & \multirow[b]{2}{*}{$<0.001 *$} \\
\hline & & False answer & $61(61.0 \%)$ & $4(4.0 \%)$ & $6(6.0 \%)$ & \\
\hline
\end{tabular}

*The statistical significance level was set at $p<0.05$; Chi-Square test for trend

TABLE (8) Replantation of avulsed permanent teeth.

\begin{tabular}{|c|c|c|c|c|c|c|}
\hline \multirow[b]{2}{*}{ School Groups } & \multirow{2}{*}{\multicolumn{2}{|c|}{$\begin{array}{c}\text { "Knocked-out permanent teeth } \\
\text { should be put back to original } \\
\text { position" }\end{array}$}} & \multicolumn{3}{|c|}{ Time } & \multirow[b]{2}{*}{ p-value } \\
\hline & & & Before & Immediate after & Six months later & \\
\hline \multirow{4}{*}{ Public } & \multirow{2}{*}{ Control } & True answer & $34(34.0 \%)$ & & $34(34.0 \%)$ & \multirow{2}{*}{1.000} \\
\hline & & False answer & $66(66.0 \%)$ & & $66(66.0 \%)$ & \\
\hline & \multirow{2}{*}{ Test } & True answer & $42(42.0 \%)$ & $99(99.0 \%)$ & $93(93.0 \%)$ & \multirow{2}{*}{$<0.001 *$} \\
\hline & & False answer & $58(58.0 \%)$ & $1(1.0 \%)$ & $7(7.0 \%)$ & \\
\hline \multirow{4}{*}{ Semi Private } & \multirow{2}{*}{ Control } & True answer & $36(36.0 \%)$ & & $36(36.0 \%)$ & \multirow{2}{*}{1.000} \\
\hline & & False answer & $64(64.0 \%)$ & & $64(64.0 \%)$ & \\
\hline & \multirow{2}{*}{ Test } & True answer & $29(29.0 \%)$ & $100(100.0 \%)$ & $98(98.0 \%)$ & \multirow{2}{*}{$<0.001 *$} \\
\hline & & False answer & $71(71.0 \%)$ & $0(.0 \%)$ & $2(2.0 \%)$ & \\
\hline \multirow{4}{*}{ Private } & \multirow{2}{*}{ Control } & True answer & $29(29.0 \%)$ & & $29(29.0 \%)$ & \multirow{2}{*}{1.000} \\
\hline & & False answer & $71(71.0 \%)$ & & $71(71.0 \%)$ & \\
\hline & \multirow{2}{*}{ Test } & True answer & $39(39.0 \%)$ & $100(100.0 \%)$ & $100(100.0 \%)$ & \multirow{2}{*}{$<0.001 *$} \\
\hline & & False answer & $61(61.0 \%)$ & $0(.0 \%)$ & $0(.0 \%)$ & \\
\hline
\end{tabular}

*The statistical significance level was set at $p<0.05$; Chi-Square test for trend 
TABLE (9) Handling of avulsed permanent teeth .

\begin{tabular}{|c|c|c|c|c|c|c|}
\hline \multirow{2}{*}{ School Groups } & \multirow{2}{*}{\multicolumn{2}{|c|}{$\begin{array}{l}\text { "Avulsed permanent teeth } \\
\text { should be hold by the crown" }\end{array}$}} & \multicolumn{3}{|c|}{ Time } & \multirow{2}{*}{ p-value } \\
\hline & & & Before & Immediate after & Six months later & \\
\hline \multirow{4}{*}{ Public } & \multirow{2}{*}{ Control } & True answer & $46(46.0 \%)$ & & $46(46.0 \%)$ & \multirow{2}{*}{0.776} \\
\hline & & False answer & $54(54.0 \%)$ & & $54(54.0 \%)$ & \\
\hline & \multirow{2}{*}{ Test } & True answer & $55(55.0 \%)$ & $97(97.0 \%)$ & $95(95.0 \%)$ & \multirow{2}{*}{$<0.001 *$} \\
\hline & & False answer & $45(45.0 \%)$ & $3(3.0 \%)$ & $5(5.0 \%)$ & \\
\hline \multirow{4}{*}{ Semi Private } & \multirow{2}{*}{ Control } & True answer & $48(48.0 \%)$ & & $48(48.0 \%)$ & \multirow{2}{*}{1.000} \\
\hline & & False answer & $52(52.0 \%)$ & & $52(52.0 \%)$ & \\
\hline & \multirow{2}{*}{ Test } & True answer & $46(46.0 \%)$ & $100(100.0 \%)$ & $93(93.0 \%)$ & \multirow{2}{*}{$<0.001 *$} \\
\hline & & False answer & $54(54.0 \%)$ & $0(.0 \%)$ & $7(7.0 \%)$ & \\
\hline \multirow{4}{*}{ Private } & \multirow{2}{*}{ Control } & True answer & $43(43.0 \%)$ & & $43(43.0 \%)$ & \multirow{2}{*}{1.000} \\
\hline & & False answer & $57(57.0 \%)$ & & $57(57.0 \%)$ & \\
\hline & \multirow{2}{*}{ Test } & True answer & $52(52.0 \%)$ & $100(100.0 \%)$ & $100(100.0 \%)$ & \multirow{2}{*}{$<0.001 *$} \\
\hline & & False answer & $48(48.0 \%)$ & $0(.0 \%)$ & $0(.0 \%)$ & \\
\hline
\end{tabular}

*The statistical significance level was set at $p<0.05$; Chi-Square test for trend

TABLE (10) Immediate replantation of avulsed permanent teeth.

\begin{tabular}{|c|c|c|c|c|c|c|}
\hline \multirow{2}{*}{ School Groups } & \multirow{2}{*}{\multicolumn{2}{|c|}{ "Place Immediately" }} & \multicolumn{3}{|c|}{ Time } & \multirow{2}{*}{ p-value } \\
\hline & & & Before & Immediate after & Six months later & \\
\hline \multirow{4}{*}{ Public } & \multirow{2}{*}{ Control } & True answer & $7(7.0 \%)$ & & $8(8.0 \%)$ & \multirow{2}{*}{0.788} \\
\hline & & False answer & $93(93.0 \%)$ & & $92(92.0 \%)$ & \\
\hline & \multirow{2}{*}{ Test } & True answer & $8(8.0 \%)$ & $68(68.0 \%)$ & $67(67.0 \%)$ & \multirow{2}{*}{$<0.001^{*}$} \\
\hline & & False answer & $92(92.0 \%)$ & $32(32.0 \%)$ & $33(33.0 \%)$ & \\
\hline \multirow{4}{*}{ Semi Private } & \multirow{2}{*}{ Control } & True answer & $8(8.0 \%)$ & & $8(8.0 \%)$ & \multirow{2}{*}{1.000} \\
\hline & & False answer & $92(92.0 \%)$ & & $92(92.0 \%)$ & \\
\hline & \multirow{2}{*}{ Test } & True answer & $7(7.0 \%)$ & $98(98.0 \%)$ & $95(95.0 \%)$ & \multirow{2}{*}{$<0.001 *$} \\
\hline & & False answer & $93(93.0 \%)$ & $2(2.0 \%)$ & $5(5.0 \%)$ & \\
\hline \multirow{4}{*}{ Private } & \multirow{2}{*}{ Control } & True answer & $6(6.0 \%)$ & & $6(6.0 \%)$ & \multirow{2}{*}{1.000} \\
\hline & & False answer & $94(94.0 \%)$ & & $94(94.0 \%)$ & \\
\hline & \multirow{2}{*}{ Test } & True answer & $8(8.0 \%)$ & $87(87.0 \%)$ & $87(87.0 \%)$ & \multirow{2}{*}{$<0.001^{*}$} \\
\hline & & False answer & $92(92.0 \%)$ & $13(13.0 \%)$ & $13(13.0 \%)$ & \\
\hline
\end{tabular}

*The statistical significance level was set at $p<0.05 ;$ Chi-Square test for trend 
TABLE (11) Delayed replantation of avulsed permanent teeth.

\begin{tabular}{|c|c|c|c|c|c|c|}
\hline \multirow{2}{*}{$\begin{array}{l}\text { School } \\
\text { Groups }\end{array}$} & \multirow{2}{*}{\multicolumn{2}{|c|}{$\begin{array}{l}\text { Store it in a proper medium and } \\
\text { seek dental clinic immediately }\end{array}$}} & \multicolumn{3}{|c|}{ Time } & \multirow{2}{*}{ p-value } \\
\hline & & & Before & Immediate after & Six months later & \\
\hline \multirow{4}{*}{ Public } & \multirow{2}{*}{ Control } & True answer & $47(47.0 \%)$ & & $41(41.0 \%)$ & \multirow{2}{*}{0.393} \\
\hline & & False answer & $53(53.0 \%)$ & & $59(59.0 \%)$ & \\
\hline & \multirow{2}{*}{ Test } & True answer & $36(36.0 \%)$ & $69(69.0 \%)$ & $67(67.0 \%)$ & \multirow{2}{*}{$<0.001 *$} \\
\hline & & False answer & $64(64.0 \%)$ & $31(31.0 \%)$ & $33(33.0 \%)$ & \\
\hline \multirow{4}{*}{ Semi Private } & \multirow{2}{*}{ Control } & True answer & $31(31.0 \%)$ & & $31(31.0 \%)$ & \multirow{2}{*}{1.000} \\
\hline & & False answer & $69(69.0 \%)$ & & $69(69.0 \%)$ & \\
\hline & \multirow{2}{*}{ Test } & True answer & $32(32.0 \%)$ & $98(98.0 \%)$ & $95(95.0 \%)$ & \multirow{2}{*}{$<0.001^{*}$} \\
\hline & & False answer & $68(68.0 \%)$ & $2(2.0 \%)$ & $5(5.0 \%)$ & \\
\hline \multirow{4}{*}{ Private } & \multirow{2}{*}{ Control } & True answer & $45(45.0 \%)$ & & $45(45.0 \%)$ & \multirow{2}{*}{1.000} \\
\hline & & False answer & $55(55.0 \%)$ & & $55(55.0 \%)$ & \\
\hline & \multirow{2}{*}{ Test } & True answer & $29(29.0 \%)$ & $87(87.0 \%)$ & $87(87.0 \%)$ & \multirow{2}{*}{$<0.001 *$} \\
\hline & & False answer & $71(71.0 \%)$ & $13(13.0 \%)$ & $13(13.0 \%)$ & \\
\hline
\end{tabular}

*The statistical significance level was set at $p<0.05$; Chi-Square test for trend

TABLE (12) Storage medium.

\begin{tabular}{|c|c|c|c|c|c|c|}
\hline \multirow{2}{*}{ Schools } & \multirow{2}{*}{\multicolumn{2}{|c|}{ Medium for storing the avulsed tooth }} & \multicolumn{3}{|c|}{ Time } & \multirow{2}{*}{-p-value } \\
\hline & & & Before & During & After & \\
\hline \multirow{4}{*}{ Public } & \multirow{2}{*}{ Control } & True answers & $13(13.0 \%)$ & & $14(14.0 \%)$ & \multirow{2}{*}{0.836} \\
\hline & & False answers & $87(87.0 \%)$ & & $86(86.0 \%)$ & \\
\hline & \multirow{2}{*}{ Test } & True answers & $29(29.0 \%)$ & $97(97.0 \%)$ & $99(99.0 \%)$ & \multirow{2}{*}{$<0.001^{*}$} \\
\hline & & False answers & $71(71.0 \%)$ & $3(3.0 \%)$ & $1(1.0 \%)$ & \\
\hline \multirow{4}{*}{ Semi Private } & \multirow{2}{*}{ Control } & True answer & $20(20.0 \%)$ & & $31(31.0 \%)$ & \multirow{2}{*}{0.104} \\
\hline & & False answer & $80(80.0 \%)$ & & $69(69.0 \%)$ & \\
\hline & \multirow{2}{*}{ Test } & True answer & $15(15.0 \%)$ & $100(100.0 \%)$ & $100(100.0 \%)$ & \multirow{2}{*}{$<0.001 *$} \\
\hline & & False answer & $85(85.0 \%)$ & $0(.0 \%)$ & $0(.0 \%)$ & \\
\hline \multirow{4}{*}{ Private } & \multirow{2}{*}{ Control } & True answer & $22(22.0 \%)$ & & $22(22.0 \%)$ & \multirow{2}{*}{1.000} \\
\hline & & False answer & $78(78.0 \%)$ & & $78(78.0 \%)$ & \\
\hline & \multirow{2}{*}{ Test } & True answer & $28(28.0 \%)$ & $100(100.0 \%)$ & $100(100.0 \%)$ & \multirow{2}{*}{$<0.001^{*}$} \\
\hline & & False answer & $72(72.0 \%)$ & $0(.0 \%)$ & $0(.0 \%)$ & \\
\hline
\end{tabular}

*The statistical significance level was set at $p<0.05 ;$ Chi-Square test for trend 
TABLE (13) Knowledge regarding mouth guards according school groups.

\begin{tabular}{|c|c|c|c|c|c|c|}
\hline \multirow{2}{*}{ School Groups } & \multirow{2}{*}{\multicolumn{2}{|c|}{$\begin{array}{l}\text { Have you heard of anything that } \\
\text { can protect teeth while sports }\end{array}$}} & \multicolumn{3}{|c|}{ Time } & \multirow{2}{*}{ p-value } \\
\hline & & & Before & Immediate after & Six months later & \\
\hline \multirow{4}{*}{ Public } & \multirow{2}{*}{ Control } & True answer & $66(66.0 \%)$ & & $69(69.0 \%)$ & \multirow{2}{*}{0.651} \\
\hline & & False answer & $34(34.0 \%)$ & & $31(31.0 \%)$ & \\
\hline & \multirow{2}{*}{ Test } & True answer & $69(69.0 \%)$ & $100(100.0 \%)$ & $100(100.0 \%)$ & \multirow{2}{*}{$<0.001^{*}$} \\
\hline & & False answer & $31(31.0 \%)$ & $0(.0 \%)$ & $0(.0 \%)$ & \\
\hline \multirow{4}{*}{ Semi Private } & \multirow{2}{*}{ Control } & True answer & $73(73.0 \%)$ & & $82(82.8 \%)$ & \multirow{2}{*}{0.124} \\
\hline & & False answer & $27(27.0 \%)$ & & $17(17.2 \%)$ & \\
\hline & \multirow{2}{*}{ Test } & True answer & $53(53.0 \%)$ & $100(100.0 \%)$ & $100(100.0 \%)$ & \multirow{2}{*}{$<0.001 *$} \\
\hline & & False answer & $47(47.0 \%)$ & $0(.0 \%)$ & $0(.0 \%)$ & \\
\hline \multirow{4}{*}{ Private } & \multirow[b]{2}{*}{ Control } & True answer & $68(68.0 \%)$ & & $76(76.0 \%)$ & \multirow{2}{*}{0.208} \\
\hline & & False answer & $32(32.0 \%)$ & & $24(24.0 \%)$ & \\
\hline & \multirow{2}{*}{ Test } & True answer & $74(74.0 \%)$ & $100(100.0 \%)$ & $100(100.0 \%)$ & \multirow{2}{*}{$<0.001 *$} \\
\hline & & False answer & $26(26.0 \%)$ & $0(.0 \%)$ & $0(.0 \%)$ & \\
\hline
\end{tabular}

*The statistical significance level was set at p<0.05; Chi-Square test for trend

\section{DISCUSSION}

In response to the World Health Organization (WHO) guidelines in the year 1978, many countries utilized school teachers as health education promoters by increasing their knowledge in oral health and disease. However, despite the willingness to impart general oral health education, they seem to lack formal basic training in oral health matters such as emergency management of DTI, which will hinder the effectiveness of teacher's role in promoting oral health ${ }^{(25)}$.

Dental Traumatic Injuries are considered a public health problem and if not treated timely and appropriately, they will not only cause disability or loss of teeth, but also will have a negative impact on the quality of life of the individual. Primary and permanent anterior teeth are not only important for aesthetics but also essential for phonetics, mastication, integrity of supporting tissues, psychological, physical and mental wellbeing $^{(22)}$. The International Association of
Dental Traumatology and American Academy of Pediatric Dentistry acknowledge that DTI could have improved outcomes if the public was aware of first aid measures and the need to seek immediate treatment ${ }^{(26)}$. Emergency treatment of DTI at the site of the accident is particularly important since an initial mismanagement will directly be reflected in the subsequent treatment, which can be complicated and lengthy; thus affecting the prognosis of the case and the patient's wellbeing.

More than $20 \%$ of school aged children are reported to be affected by dental trauma ${ }^{(27)}$. For this reason, we centred our study in school teachers who are usually the first individuals to respond to the traumatic incident in schools.

Actually, in Lebanon, no attempt has been made by the government or any dental organization to educate teachers by providing them information regarding DTI. Consequently, the purpose of the study was to assess the effectiveness of educational message on the knowledge of teachers regarding 
DTI in schools of Beirut-Lebanon for better practice and prognosis of these cases.

This study was carried out between January and July,2016inthecityofBeirut-Lebanon.Itwasdecided to work with teachers from public, semiprivate and private schools. A cluster randomized controlled trial, commonly used in school-based evaluations of educational interventions, was designed. This cluster randomized design appears to have begun back in 1940 with Lindquist's book on "Methods in education research in schools" and has been defined as a comparative study in which the units randomized are groups whose members have an identifiable feature in common ${ }^{(28,29)}$. Two-hundred teachers per group (public, semi private and private) were randomly selected and a questionnaire was applied to assess their knowledge regarding the topic prior to the implementation of the educational program. Consequently, a total of 600 teachers were included in the study; half of them belonging to the test group and the other half to the control group. A similar sample size was used by Sreelakshmi et al. in $2016^{(14)}$ and Shamarao et al. in $2014^{(17)}$. Up to our knowledge, none of studies conducted compared the three school categories all together.

In the present study, a non interventional control group was included, so that we could compare any change of knowledge between the test and control groups from baseline till the end of the study after six months.

The majority of successful interventional studies have used a multifaceted approach. Based upon these findings, it was decided to combine the survey with an oral presentation in conjunction with informational brochures and posters. The most commonly used methods of oral presentation are seminars and lectures. A lecture using Power Point presentation performed in this study provided a good opportunity for direct contact with the teachers, thus giving a more dynamic discussion and immediate feedback in order to respond to any doubt they may have, as well as enhancing their comprehension about the topic to ensure that the information being delivered is understood ${ }^{(24)}$. After 30 minutes of lecture, followed by questions and answers, it was possible to motivate the teachers by improving their knowledge and practice regarding DTI.

As complementary educational tools, brochures were given to teachers and also a poster per school in order to increase the power of the educational message and to spread the information to more teachers, parents and any other children guardians. Displaying educational posters in classrooms is a practical and effective means to improve knowledge of DTI ${ }^{(23)}$.

Chi square test for trend was carried out to compare the knowledge level over a period of time (before and after the implementation of the educational message)

Due to the approvals given by the ministries to undertake the study, teachers belonging to public schools were the most helpful and supportive with the study. On the other hand, private schools were the least cooperative since they considered that it was not their job to learn about DTI because they have a nurse at the school. However, once they accepted, they showed great curiosity and interest.

Despite the fact that teachers' level of awareness concerning DTI before the implementation of the educational program was noticeably inadequate, and regardless of how unsatisfied they felt with their lack of knowledge; they exhibited a positive attitude towards receiving more information regarding emergency management of these injuries. The positive attitude of the teachers reflected in our study is similar to the response observed in a survey done by Shamarao et al. in $2014^{(17)}$.

Immediately after the implementation of the educational message, there was a significant improvement in the knowledge and practice of teachers belonging to the test group. Even six months later, an improvement of $90 \%$ and above was still noticeable in some areas. While, in the control group there was no knowledge acquisition 
in the majority of the questions; similar to a study conducted by Ghadimi et al. in $2014^{(21)}$. However, a slight increase in the correct answers was observed in questions regarding storage medium and mouth guards, which was related to the fact that the questionnaire itself raised their curiosity level of knowing more and even some of the teachers used to discuss these topics with each other after the completion of the survey.

In private schools, there were significantly more females than males and also more teachers with a superior educational level compared to public and semi private schools, which is related to the fact that private schools have particular recruitment policies that pursue specific requisites for teachers requiring university degree. After the analysis of the first questionnaire, it was observed that the gender, level of education of teachers and the school group they belonged to (private, semi private and public) were not directly associated with the understanding or the knowledge they had regarding DTI. This is probably because very little or no information about this topic had been previously given to most of them. However, six months later, a more noticeable decrease was observed in the correct answers of public school teachers in questions regarding the management of DTI such as fractures, displacement, avulsion, immediate and delayed replantation; which is in accordance with a study done in Kuwait by Al-Asfour et al. in $2008^{(24)}$. Conversely, the lowest decrease was detected in private school teachers which could be directly related to their level of education. Similarly, there are studies showing that the level of education has a positive impact on teachers' knowledge about topics that do not belong to their specific area of study, including dental trauma ${ }^{(19)}$.

This acts as an indicator that teachers may need more frequent reinforcement within shorter periods of time. Most likely the information has to be repeated before any long standing effect can be noted. Knowledge retention is critically important in emergency management of DTI education as we attempt to teach concepts that participants may use and apply at any time in the future.

It can be said that a combination of the questionnaires, a power point presentation, and informational brochures and posters provided positive and successful results. Even after 6 months, the level of knowledge concerning DTI among these teachers (belonging to the test group) was very satisfactory. Similar studies conducted by Grewal (2015), Ghadimi (2014), Pujita (2013), Arikan (2012) and McIntyre et al. (2008); assessed the knowledge of elementary school staff members regarding management of DTI before and after educational intervention. Also it was found that a combination of lectures and brochures significantly enhance the knowledge among staff, and the improvement persisted over time.

This study has a few limitations. For instance, there is no data on whether participants were parents of young children or not, which could be a confounding factor. Also, the subject that each participant was responsible for teaching was not recorded (e.g. biology, physical education, geography).

Intuitive guessing has been previously suggested regarding this topic when employing questionnaires which could be a possible limitation of this study. ${ }^{(30)}$

This study was conducted only in Beirut by taking a representative sample of the teachers and no other cities of Lebanon were included in the trial.

\section{CONCLUSION}

The lack of knowledge of teachers regarding DTI before the implementation of the educational message was confirmed.

Within the limitation of this study we can conclude that, the implementation of a health educational program regarding DTI using more than one modality, proved to be effective in improving the knowledge of school teachers who are the first individuals to face dental trauma in schools. 


\section{RECOMMENDATIONS}

1. Dentists as health educators and authorities in the country, as Ministry of Health and Ministry of Education, must work together to reach more school teachers for the continuous dissemination of knowledge with an emphasis on the management of DTI.

2. More studies should be conducted in other regions of Lebanon to assess teachers' knowledge as well and to widen the awareness level, in addition to conducting direct interviews with the target group.

3. To ensure accessibility to proper information; brochures and posters describing the emergency procedures in traumatic situations should be displayed in high-risk environments, such as schools, so that appropriate action can be taken during such events.

\section{REFERENCES}

1. Sprod A, Anderson R \& Treasure E. Effective oral health promotion: Literature Review. 1996.

2. Abdellatif AM, Hegazy SA. Knowledge of emergency management of avulsed teeth among a sample of Egyptian parents. J Adv Res 2011;2:157-62.

3. Cortes MI, Marcenes W, Sheiham A. Impact of traumatic injuries to the permanent teeth on the oral healthrelated quality of life in 12-14-yearold children. Community Dent Oral Epidemiol 2002; 30:193-8.

4. Diangelis AJ, Andreasen JO, Ebeleseder KA, Kenny DJ, Trope M, Sigurdsson A, et al. International Association of Dental Traumatology guidelines for the management of traumatic dental injuries: 1. Fractures and luxations of permanent teeth. Dent Traumatol 2012;28:2-12.

5. Glendor U. Epidemiology of traumatic dental injuries-a 12 year review of the literature. Dent Traumatol 2008;24: 603-611

6. Andreasen JO, Andreasen FM, Andersson L. Textbook and colour atlas of traumatic injuries to the teeth. 4 th edn. Oxford, Blackwell Munksgaard; 2007. P. 383-427.

7. Ankola A, Hebbal M, Sharma R, Nayak S. Traumatic dental injuries in primary school children of South India - a report from district-wide oral health survey. Dental Traumatology 2013; 29: 134-138.

8. Mesgarzadeh AH, Shahamfar M, Hefzollesan A. Evaluating knowledge and attitudes of elementary school teachers on emergency management of traumatic dental injuries: A study in an Iranian urban area. Oral Health Prev Dent 2009;7(3):297-308.

9. Dame-Teixeira N, Alves LS, Susin C, Maltz M. Traumatic dental injury among 12-year-old South Brazilian schoolchildren: prevalence, severity, and risk indicators. Dent Traumatol 2013;29:52-58.

10. Marchiori EC, Santos SE, Asprino L, Moraes M, Moreira RW. Occurrence of dental avulsion and associated injuries in patients with facial trauma over a 9-year period. Oral Maxillofac Surg. 2013;17(2):119-26.

11. Bonini GAVC, Marcenes W, Oliveira LB, Sheiham A, Bonecker M. Trends in the prevalence of traumatic dental injuries in Brazilian preschool children. Dent Traumatol. 2009;25(6):594-8.

12. Kane AW, Babacar T, Moustapha D, Malick M, Mouhamed S, Yves B. Attitude and knowledge of primary school teachers of initial management of dental trauma. JOHR 2011;2(2):51- 54.

13. Tzigkounakis V, Merglova V. Attitude of Pilsen primary school teachers in dental traumas. Dental Traumatology; 2008; 24: 528-531.

14. Sreelakshmi N, Rajendra Reddy E, Thabitha Rani S, Rajesh Aduri, Vinay Kumar L, Mahita P. Assessment of Knowledge and Attitude among Public and Private School Teachers in Urban and Rural Areas towards Management of Traumatic Dental Injuries. Journal of International Oral Health 2016; 8(3):344-350.

15. Grewal N, Shangdiar GD, Samita G. Efficacy of a comprehensive dental education program regarding management of avulsed permanent teeth as a valid indicator of increased success rate of treatment of avulsion in a North Indian population. Contemp Clin Dent 2015;6:477-82.

16. Singh M, Ingle NA, Kaur N, Yadav P. Evaluation of knowledge and attitude of school teachers about emergency management of traumatic dental injury. J Int Soc Prevent Communit Dent 2015;5:108-13.

17. Shamarao S, Jain J, Ajagannanavar SL, Haridas R, Tikare S, Kalappa AA. Knowledge and attitude regarding management of tooth avulsion injuries among school teachers in rural India. J Int Soc Prevent Communit Dent 2014;4:S44-8. 
18. Arikan V, Sonmez H. Knowledge level of primary school teachers regarding traumatic dental injuries and their emergency management before and after receiving an informative leaflet. Dent Traumatol. 2012;28(2):101-7.

19. Feldens EG, Feldens CA, Kramer PF, da Silva KG, Munari $\mathrm{CC}$, Brei VA. Understanding school teacher's knowledge regarding dental trauma: a basis for future interventions. Dent Traumatol 2010;26:158-163.

20. Raoof M, Shokouhinejad N, Izadi A, Nourzadeh M, Afkham A, Forghani FR, et al. Longterm effect of an educational intervention regarding dental trauma first aid: A phase II study. Dent Traumatol 2014;30:296301.

21. Ghadimi S, Seraj B, Keshavarz H, Shamshiri AR, Abiri R. The Effect of Using an Educational Poster on Elementary School Health Teachers' Knowledge of Emergency Management of Traumatic Dental Injuries. Journal of Dentistry, Tehran University of Medical Sciences, Tehran, Iran 2014; Vol. 11, No. 6

22. Pujita C, Nuvvula S, Shilpa G, Nirmala S, Yamini V. Informative promotional outcome on school teachers' knowledge about emergency management of dental trauma. J Conserv Dent 2013;16(1):21-7.

23. Young C, Wong KY, Cheung LK. Effectiveness of Educational Poster on Knowledge of Emergency Management of Dental Trauma - Part 2: Cluster Randomised Controlled Trial for Secondary School Students. 2014. PLoS ONE 9(8): e101972. doi:10.1371/journal.pone.0101972.
24. Al-Asfour A, Andersson L. The effect of a leaf et given to parents for first aid measures after tooth avulsion. Dent Traumatol 2008; 24:515-21.

25. Sae Sankar AJ, Sreedevi E, Suresh Babu M, Naveen V, Rajavardhan K. School teacher's knowledge regarding dental health. Indian J Dent Sci 2013;5:1558.

26. Zakirulla M, Togoo RA, Yaseen SM, Al-Shehri DA, AlGhamdi AS, Al-hafed MS, et al. Knowledge and attitude of Saudi Arabian school teachers with regards to emergency management of dental trauma. Int J Clin Dent Sci 2011;2(2):25-9.

27. Ankola A, Hebbal M, Sharma R, Nayak S. Traumatic dental injuries in primary school children of South India - a report from district-wide oral health survey. Dental Traumatology; 2013; 29: 134-138.

28. Stockwell MS, Catallozzi M, Camargo S, Ramakrishnan R, Holleran S, Findley SE, Kukafka R, Hofstetter AM, Fernandez N, Vawdrey DK. Registry-linked electronic influenza vaccine provider reminders: a cluster-crossover trial. Pediatrics. 2015 Jan; 2015; 135 (1):e75-82.

29. Lindquist EF. Statistical analysis in educational research. Boston: Houghton Mifflin. 1940.

30. McIntyre JD, Lee JY, Trope M, Vann WF Jr. Elementary school staff knowledge about dental injuries. Dent Traumatol 2008; 24(3):289-98. 